\title{
Culture, Obesity Stereotypes, Self-Esteem, and the "Thin Ideal": A Social Identity Perspective
}

\author{
Paul A. Klaczynski, ${ }^{1}$ Kristen W. Goold, ${ }^{2}$ and Jeffrey J. Mudry ${ }^{3}$
}

Received November 26, 2003; revised February 5, 2003; accepted January 20, 2004

Recent concerns with the increasing prevalence of overweight and obesity among children and adults indicate the need to better understand the psychosocial correlates of weight. We examined the relationships among negative stereotypes of obesity, "thin ideal" beliefs, perceptions of the causes of obesity and of control over weight, body esteem, and global self-esteem. A negative correlation between beliefs in control over one's weight and self-esteem was mediated by both negative attitudes toward obesity and thin idealization. Additionally, body esteem and gender were related but this relationship was mediated by beliefs in control over weight and valuation of the thin ideal. Central to the theoretical foundation of this research, however, was the observed negative correlation between negative attitudes toward obesity and self-esteem. This relationship was mediated primarily by the belief that obesity is caused by personality shortcomings. This last finding is explained from a social identity perspective.

KEY WORDS: obesity stereotypes; social identity; thin idealization; personal control over weight.

\section{INTRODUCTION}

Movement from adolescence into adulthood is characterized by not only such social and economic transitions as graduation from high school, financial independence, and entry into the full-time work force but also by increased health risks (Steinberg, 2002). Indeed, a considerable body of research has documented normative changes that characterize the development of adolescents and youths (e.g., Peterson, 1988) and a second corpus of work has examined numerous aspects of nonnormative development (e.g., Jessor et al., 1995; Osgood et al., 1996), such as delinquency, substance abuse, unplanned preg-

\footnotetext{
${ }^{1}$ Associate Professor at The Pennsylvania State University. Received his Ph.D. life-span developmental psychology from West Virginia University in 1989. His major interests are adolescent decision making and reasoning, stereotype development, and the links among ethnicity, identity, and obesity stereotypes. To whom correspondence should be addressed at the Pennsylvania State University, University Park, Pennsylvania; e-mail: pak21@psu.edu.

${ }^{2}$ Received her undergraduate degree in psychology from The Pennsylvania State University and is interested in adolescent development and counseling.

${ }^{3}$ An undergraduate in The Pennsylvania State University's Psychology Department. His interests are in stereotyping, adolescence, and counseling.
}

nancy, and disturbed eating patterns (e.g., anorexia, bulimia; e.g., Archibald et al., 1999). Conspicuously absent from both literatures is research on obesity, a problem that has become increasingly common in modern youths and adolescents. Although studies documenting relationships between weight and various aspects of self-perceptions (e.g., self-esteem; e.g., Mendelson et al., 1996) does exist, almost nonexistent is work exploring the relationships among weight, self-perceptions, and other-perceptions (particularly, stereotypes of obesity). Yet such research is critical if we are to understand possible links of weight status and weight-related beliefs to numerous psychosocial phenomena (see Bell and Morgan, 2000). For instance, beliefs about obesity (i.e., beliefs about its causes, correlates, and consequents) may lead to discrimination, prejudice, self-stigmatization, and poor peer relationships. Because the transition from high school to college is often stressful (Boyer, 1986), young overweight adults may be particularly vulnerable to the adverse effects of their peers' perceptions. The research presented herein was undertaken as an initial attempt to illuminate some of the relationships among youths' weight, their self-perceptions, and their beliefs about obesity.

Concerns with the rising rates of obesity and overweight have been voiced with increasing frequency by 
pediatricians, health professionals, and the media. Recent epidemiological data indicate that the risks of obesity for 6- to 11-year-old children and 12- to 19-year-old adolescents have increased from approximately $8 \%$ and $6 \%$ in the late 1970 s to $14 \%$ and $13 \%$, respectively, at the beginning of the new millennium (Gable and Lutz, 2000; Nestle, 2002). These risks are even greater among adults, as about $33 \%$ of American adults are overweight and an additional 25\% are obese (CDC, 2002; Nestle, 2002). The transition from adolescence to adulthood is thus marked by changes in economic status, relationships, independence, and risks for overweight and obesity.

Two questions served as foundations for the present research. First, the extent to which adolescents' stereotypes of and attitudes toward obesity could be predicted from self-esteem, internalization of the premium Western societies place on thinness, and beliefs about the causes of obesity was explored. Second, we examined whether youths' self-esteem could be predicted from perceptions of control over weight, attitudes toward obesity, and value placed on the "thin ideal" that saturates youth culture.

\section{Cultural Belief Systems, Self-esteem, and Attitudes Toward Obesity}

A prominent feature of the belief systems that pervade modern postindustrial societies, and a characteristic that has long characterized American culture, is the view that accomplishments and failures-whether academic, economic, or relational-are outcomes of personal motivations (of the lack thereof) to be successful (Simmons and Rosenberg, 1971; Turner, 1960). Although some variation in this and related beliefs can be found within most cultures, at least in the United States, Caucasian adolescents and young adults from a variety of social, economic, and educational backgrounds generally perceive individual achievements to be far more determined by effort and ability than by environmental forces (Klaczynski, 1991), such as parental support and economic background.

This emphasis on individualism has implications for the social lives of overweight and obese children and adults (Crandall et al., 2001; Crystal et al., 2000). Women and girls in postmodern societies are bombarded with messages from the media, parents, and peers that the ideal body is one that is almost impossibly thin. That is, in advertisements of products ranging from make-up to beer, the image of the "successful" and attractive woman is she who looks most like the dangerously thin models commonly featured in such magazines as Seventeen, Vogue, and Cosmopolitan (Thompson and Stice, 2001). Although images of success are more variable for young men, it is still the case that the successful man - and the boy likely to be popular with his peers-is a muscular, but nonetheless slim - athlete with an unrealistically narrow waist.

The Western emphasis on individualism suggests that youths will view their peers' weight and, indeed, their own weight as outcomes of personal efforts and failures to the achieve the "thin ideal" (see also Pierce and Wardle, 1997; Quinn and Crocker, 1999). Those who fail to cultivate a "body for success" are, in this view, likely to be perceived as weak-willed, as lacking the skills and motivations needed not only to triumph over their bodies but also for success in other domains, and as possessing characteristics that are generally undesirable (Crystal et al., 1997; Quinn and Crocker, 1999). The more individuals are believed to deviate from our culture's body ideals, the more likely they are to be perceived (by self and peers) as personal failures, and the lower their physical and social attractiveness (Bell and Morgan, 2000; Crandall et al., 2001; Thompson and Stice, 2001).

Research with children and adults generally supports the hypothesis that obese individuals are negatively stigmatized. A consistent finding among pediatricians (e.g., Bell and Morgan, 2000) and psychologists (e.g., Crocker et al., 1993; Lerner and Korn, 1972) is that the obese are more negatively stigmatized than almost all other social groups. Evidence from a variety of methodological traditions has shown that ethnic minorities, women, people in wheelchairs, amputees, and children and adults with facial disfigurements are all viewed more positively (e.g., friendlier, more likely to succeed, etc.) than obese persons (Staffieri, 1967; Wiener et al., 1988; Wing and Jeffrey, 1999). Perhaps the most critical factor distinguishing obesity from social categories like those listed above is that the obese are more likely to be held personally accountable for their weight (Crandall, 1994; Crandall and Biernat, 1990; Wiener et al., 1988).

Clearly, however, not all youths denigrate those who are overweight (Crocker et al., 1993). In this paper, we explore the possibility that individual differences in beliefs about the perceived potential of the obese to change their physical and social standing, the extent to which the "thin ideal" has been internalized and accepted, and self-esteem predict the tendency to hold obese individuals in low regard. Our first prediction, derived from the argument in the previous paragraph, was that negative attitudes toward the obese would be related to beliefs about the causes of obesity. Specifically, those who believe that obesity is the result of lack of impulse control and other personal shortcomings should be more likely than those who attribute obesity to external causes (e.g., medical, hereditary, environmental) to express negative feelings toward and stereotypes of obese individuals. 
Other hypotheses were based on social identity theory (Tajfel, 1982; Tajfel and Turner, 1979). According to social identity theorists, self-esteem is partly determined by the ability of members of a social group or category (e.g., average weight people) to see their group as superior to other groups (e.g., obese people). The higher a group's status, the more its members stand to gain by affiliating with it. Thus, information about members of an "in-group" is typically evaluated more positively than information about "out-groups" (Hewstone et al., 1982, 1993; Pettigrew, 1979; Simon and Brown, 1987), even when that information is logically identical (Klaczynski, 2000; Klaczynski and Aneja, 2002).

Individuals who most closely identify with an ingroup and who most strongly uphold the beliefs and values of the in-group are more likely than those less strongly affiliated with the group to derogate out-groups. Why should some people identify more closely with a high status group than other people? One argument made by social identity theorists is that people who feel most closely attached to an in-group are those whose social or emotional status indicates that they would benefit from affiliation. One of the clearest indicators that a person will be higher than his or her peers in this need for affiliation is low self-esteem (see Baumeister and Leary, 1995). Particularly when a group has a high social standing, individuals with low self-esteem should seek membership benefits more often and should identify more closely with the ingroup's values than high self-esteem individuals (Bigler et al., 1997). Hence, our second prediction was that the lower a person's self-esteem, the greater their acceptance of the beliefs that life success and thinness are intimately related.

One means of increasing the value and salience of group membership is to portray the group more positively than other groups. Consequently, the promotion of an in-group and its values often comes at the expense of out-groups. That is, the uniqueness of an in-group can be established and its status can be elevated by derogating out-groups not believed to share the group's beliefs (Hewstone et al., 1982; Tajfel and Turner, 1981). This reasoning led to our third prediction: That self-esteem among nonobese individuals would correlate negatively with derogatory beliefs about and attitudes toward obese persons.

We have thus far suggested three simple correlations between: self-esteem and in-group values (i.e., the "thin ideal"), self-esteem and negative stereotypes of the obese, and beliefs about the causes of obesity (i.e., as resulting from personality "deficits") and negative characterizations of the obese. However, individualistic beliefs permeate virtually all aspects of social life. Portraits of the "to be thin, is to be in" ideology are ubiquitous in Western cultures. However, there are individual differences in the extent to which youths subscribe to this belief system. This suggests the following mediational hypothesis: We expected that the relationship between self-esteem and negative beliefs about obesity would be mediated by the extent to which obesity was attributed to personal failures (on the part of overweight people) to exert self-control and by the extent to which the "thin ideal" is accepted. Further, because beliefs in the "thin ideal" are likely related to beliefs that weight is in the province of personal control, not only should internal causal attributions correlate positively with acceptance of the "thin ideal," but the former belief system should be the more powerful mediator of the self-esteem/negative attitudes toward obesity relationship.

\section{Perceptions of Control Over Weight and Self-esteem}

The foregoing discussion focused on processes that may be involved in the creation of prejudices toward obese individuals. However, these same prejudices may affect self-esteem adversely. Specifically, the individualistic assumptions about personal success and failure that saturate Western societies - coupled media messages that individuals should "take control of their bodies" and with actual evidence that at least some people can control their weight - could lead youths to believe that weight is controllable through personal actions. If an individual internalizes this belief, but (perhaps because of a history of fruitless dieting) believes that his or her own weight cannot be controlled, self-esteem may suffer (i.e., "In this important domain, I should have control, but I do not. I am, therefore, a failure in this respect"). It is unlikely, however, that perceptions of personal control over weight directly affect self-esteem. Instead, perceived lack of control over weight should affect self-esteem only to the extent that individuals devalue overweight persons and have internalized beliefs about the value of thinness. Thus, perceiving that they have no control over their weight, individuals may devalue themselves because (a) they believe that they may fall into a devalued social category, and that there is little that can be done to prevent such a fall and (b) they hold themselves to an unrealistically high standard that they lack the skill, willpower, etc. to attain. As the basis of this reasoning, we hypothesized that the relationship between perceptions of control over one's weight and self-esteem would be mediated by valuation of the thin ideal and devaluation of obese persons. 


\section{Body Esteem, the Thin Ideal, and Perceptions of Control Over Weight,}

Numerous arguments for a negative relationship between overweight and self-esteem have been forwarded, but researchers have found somewhat mixed support for this relationship. Although a recent meta-analysis (Miller and Downey, 1999) showed that a relationship does exist, the strength of this relationship varies with SES, ethnicity, age (although negative correlations between weight and self-esteem have been reported for children, adolescents, and college students) and gender. We reexamined the weight-self-esteem relationship in this study and focused specifically on the relationship between weight and body esteem.

Particularly among Caucasian adolescents and adults (and even more so for women than for men), self-esteem and body self-esteem are clearly correlated (Mendelson et al., 1996). As Harter $(1985,1993)$ and others (e.g., Mendelson et al., 1996) have argued, global self-esteem derives from self-esteem in specific domains. The more a culture emphasizes the importance of specific domains for life success, the more self-esteem in those domains will affect global self-esteem.

Prior research has shown clear associations between body esteem and weight and between body esteem and gender. Because Western societies place a premium on fitness and thinness as important determinants of interpersonal and economic success, we hypothesized that internalization of the thin ideal would mediate both the relationship between weight and body esteem and the relationship between gender and body esteem. A second potential mediator is related to the tendency of people in Western societies to believe that body "success," like success in other domains, is personally controllable. Those who accept this belief, but who believe that they cannot control their own weight (i.e., "I should be in control of my weight, but I don't") should have lower body esteem than people who believe that they can control their weight. Our final hypothesis, then, was that the gender->body esteem and weight- $>$ body esteem relationships would also by mediated by the belief that one's weight is controllable/uncontrollable.

\section{METHODS}

\section{Participants}

A total of 107 male $(n=17)$ and female $(n=90)$ undergraduate students from a large northeastern university were recruited for participation from introductory psy- chology classes (mean age $=19.23$ years; $S D=1.11$ years). All participants received course credit for participating. The measures described below were administered to small groups of 2-5 participants.

\section{Measures}

At the outset of each session, participants indicated their height and weight, which was then used to calculate body mass index (BMI). Although self-reported weight is often an underestimation of actual weight (and, among some underweight women, sometimes an overestimate of actual weight), self-report is likely to restrict the range of weights in a study and, as a consequence, make analyses more conservative than when weight is actually measured. Further evidence for appropriateness of weight estimates as proxies for actual weight is provided in Table III, which shows that BMI scores correlated with other variables in the predicted direction.

Each participant was administered a battery that included measures of self-esteem, body esteem, a causes of obesity scale, beliefs about the personal control over one's weight, attitudes toward and stereotypes of obese persons, thin idealization, and verbal ability. Verbal ability was also assessed at the outset of each session; the remaining questionnaires were presented in one of three randomly determined orders.

\section{Self-Esteem}

To measure self-esteem, the Rosenberg Self-Esteem Scale (Rosenberg, 1979) was administered. This scale has been used extensively in studies of youths and adolescents and comprises 10 items (e.g., "I take a positive attitude toward myself"; 1 = strongly disagree; $4=$ strongly agree) intended to measure self-worth. Internal reliability (Cronbach's alpha) was .90.

\section{Body Esteem}

Body esteem is defined as the extent to which a person has positive feelings and beliefs about his or her physical characteristics, particularly those characteristics related to attractiveness. To measure these beliefs, the Mendelson and White (Mendelson et al., 1995, 1996) Body Esteem Scale (BES) was administered. The BES $(\alpha=0.90)$ contains 23 items (e.g., "I'm proud of my body," "I wish I looked better" (scoring reversed on second item)), each of which was rated on a 5-point scale $(1=$ strongly disagree $; 5=$ strongly agree $)$. 
Table I. Items in The Causes of Obesity Instrument

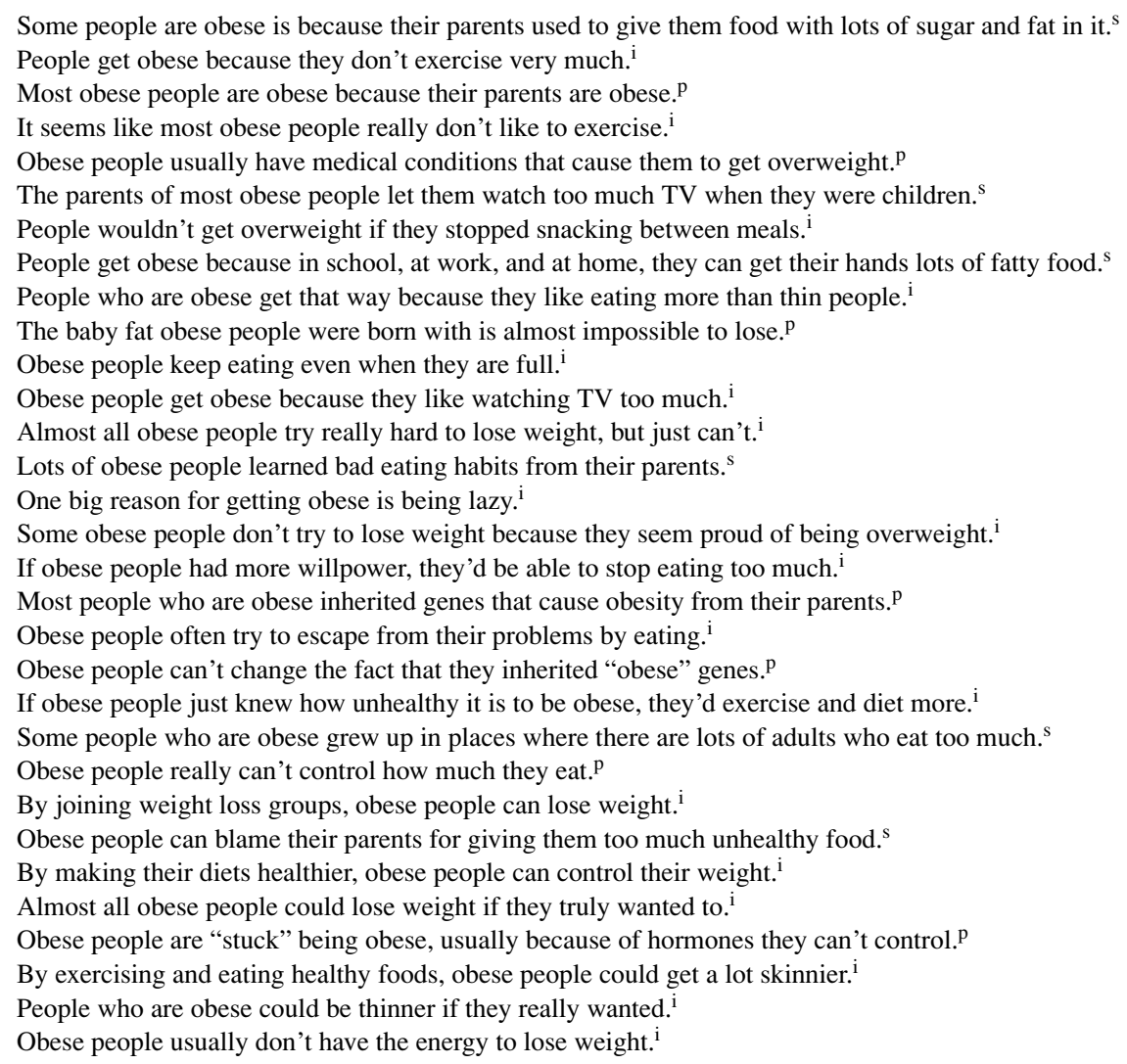

Note. The subscale to which items were assigned was first determined conceptually and then, in a pilot study, by means of a confirmatory factor analysis. On the basis of loadings in the factor analysis, some items (e.g., "Obese people usually don't have the energy to lose weight") that seemed to fit conceptually on one subscale (e.g., the PC subscale) were assigned to a different subscale (e.g., the IC scale).

${ }^{\mathrm{s}}$ Social cause subscale.

pPhysical cause subscale.

${ }^{\mathrm{i}}$ Internal cause subscale.

\section{Causes of Obesity}

The Causes of Obesity Scale, developed specifically for this research, comprises 31 items, each of which is rated on a 4-point scale $(1=$ strongly disagree; $4=$ strongly agree), and three subscales. The complete list of items is presented in Table I. The internal causes (IC) scale (18 items) assesses beliefs that obesity results from personality shortcomings and that obese people are responsible for their weight (e.g., "If obese people had more willpower, they'd stop eating too much," "It seems like most obese people don't like to exercise"). The 7-item physical causes (PC) scale assesses beliefs that obesity is a generally uncontrollable condition that is the outcome of medical and genetic mechanisms (e.g., "Obese people are stuck being obese because of hormones they can't control"). The social causes (SC) scale (6 items) measures beliefs that environmental factors are responsible for obesity (e.g., "The parents of most obese people let them watch too much TV when they were young"). Pilot research indicated that the scale had adequate internal consistency $(\alpha=.83)$ and that, after stereotypes of obesity were primed, IC scores increased (but, as expected, neither PC nor SC scores changed). In the present study, $\alpha=.79$ (internal causes, $\alpha=.84$; physical causes, $\alpha=.63$; social causes, $\alpha=.60$ ).

\section{Attitudes Toward and Stereotypes of Obesity}

Two scales were used to measure attitudes toward and stereotypes of obese people. The Antifat Attitudes Questionnaire was an adapted version of the instrument developed by Crandall (1994). The scale contains 18 items (three items from the original scale were omitted because 
they tapped into personal fears about gaining weight rather than attitudes toward obese people; 8 items, adapted from the Lewis et al. (1997) anti-fat attitudes questionnaire, were added; $\alpha=.84$ ) designed to tap negative reactions to and willingness to associate with obese people (e.g., "I really don't like fat people," "I really can't stand to look at fat people because it's too disgusting"). Items were rated on a 6-point scale $(1=$ strongly disagree; $6=$ strongly agree $)$.

The Obese Stereotypes Scale, developed for this study, consisted of 40 items $(1=$ strongly disagree $; 4=$ strongly agree) intended to measure beliefs about the personalities of obese individuals; $\alpha=.91$. The items used in the scale are presented in Table II.

Table II. Items on the Obesity Stereotypes Instrument

In my opinion, fat people are/have:

A strong sense of morality

Not very intelligent

Easily distracted

Dirty and messy

Friendly

Boastful, Brag a lot

Too dependent on others

Popular

Brave

Irresponsible

Not very much self-control

Untrustworthy

Whiny

Tattletales

Low self-esteem

Lazy

A positive outlook on life

Gossipy

Honest

Easily confused

Gloomy

Unfriendly

Loud

Don't like themselves very much

Greedy

A certain "inner strength"

Socially awkward

Think that "everyone's out to get them"

Funny

Boring to talk to

Wasteful

Nosey

Talented

Happy with TV and video games

Helpful

Self-centered

Ashamed

Lonely

Don't get annoyed very easily

Mean.

\section{Thin Idealization}

The Sociocultural Attitudes Toward Appearance Questionnaire (SATAQ; Heinberg et al., 1995) is a 15item measure of the extent to which prevailing cultural beliefs about thinness and its importance have been internalized. Each item (e.g., "Music videos that show thin women make me wish that I were thin," "Attractiveness is very important if you want to get ahead in our culture") is rated on a 5 -point scale $(1=$ disagree strongly; $5=$ agree strongly). Prior research (see Thompson and Stice, 2001) has shown that SATAQ scores predict body esteem, self-esteem, and a variety of patterns of disordered eating. In the present study, $\alpha=.88$.

\section{Control Over Weight}

The Personal Control over Weight (PCOW) Scale, designed specifically for this study, comprises 10 items designed to assess the extent to which individuals' believe that their weight, both currently (e.g., "With willpower, I can control my weight"; this item was reverse scored) and in the future (e.g., "No matter what I do, I'll always be as thin or as heavy as I am now"), can be controlled by their actions. Each item was rated on a 4-point scale $(1=$ disagree strongly; $4=$ agree strongly $)$. Total scores $(\alpha=.73)$ were reflected so that high scores indicated a participant's belief that his or her weight is difficult to control.

\section{Verbal Ability}

Prior to administration of the questionnaires, the PMA verbal meaning test (Thurstone, 1962) was administered. This test was selected to index general ability because vocabulary is the best single predictor of global intelligence scores and because the construct validity of this test has been demonstrated in several studies of adolescent and adult reasoning (see Klaczynski, 2000; Willis and Schaie, 1996). For each of 64 items, the task is to select which of four words has the same meaning as a target word. A total of 5-min were given to complete as many items as possible is a 60 -item multiple choice vocabulary test.

\section{RESULTS}

In the analyses below, correlational analyses of relationships among variables are discussed first, with a focus on those relationships that were central to our hypotheses. Next, a series of multiple regression analyses is presented. 
Table III. Zero-Order Correlations Among Measures

\begin{tabular}{|c|c|c|c|c|c|c|c|c|c|c|c|}
\hline & 2 & 3 & 4 & 5 & 6 & 7 & 8 & 9 & 10 & 11 & 12 \\
\hline 1. BMI & -0.06 & -0.42 & -0.07 & 0.03 & -0.10 & 0.33 & 0.02 & -0.06 & 0.01 & 0.07 & -0.13 \\
\hline 2. SE & & 0.66 & -0.24 & -0.20 & -0.19 & -0.30 & -0.17 & -0.07 & 0.09 & -0.04 & -0.15 \\
\hline 3. BES & & & -0.48 & -0.12 & -0.09 & -0.50 & -0.15 & -0.07 & 0.03 & -0.06 & -0.27 \\
\hline 4. SATAQ & & & & 0.15 & 0.31 & 0.29 & 0.30 & 0.20 & -0.10 & 0.08 & 0.35 \\
\hline 5. STER & & & & & 0.62 & 0.01 & 0.48 & 0.23 & 0.04 & -0.13 & -0.08 \\
\hline 6. AFA & & & & & & 0.02 & 0.49 & 0.25 & -0.16 & 0.09 & 0.03 \\
\hline 7. PCOW & & & & & & & -0.20 & 0.15 & 0.19 & 0.08 & 0.19 \\
\hline 8. INT-CAUS & & & & & & & & 0.46 & -0.18 & -0.19 & 0.19 \\
\hline 9. SOC-CAUS & & & & & & & & & -0.12 & 0.01 & 0.01 \\
\hline 10. PHY-CAUS & & & & & & & & & & -0.01 & -0.05 \\
\hline 11. VERB & & & & & & & & & & & -0.14 \\
\hline 12. GENDER $^{a}$ & & & & & & & & & & & \\
\hline
\end{tabular}

Note. Significant correlations are in bold.

${ }^{a}$ Male participants were assigned a value of 1 ; female participants were assigned a value of 2 .

Values between 0.19 and $0.24, p \leq 0.05$; values between 0.25 and $0.30, p \leq 0.01$; values above $0.30, p<0.001$.

The first regression focused on the hypothesis that the relationship between self-esteem and obesity stereotypes is mediated by beliefs that obesity results from personal shortcomings and by the internalization of cultural attitudes about thinness. The second regression was aimed at determining whether "thin ideal" beliefs and negative attitudes toward obesity can be used to explain the relationship between beliefs in personal control over one's weight and self-esteem. A third regression analysis was directed toward determining whether thinness internalization and beliefs that one's weight is controllable/uncontrollable mediated the relationship between gender and body esteem.

\section{Correlational Analyses}

Zero-order correlations among the scales and with BMI, gender, and verbal ability are presented in Table III. Although numerous correlations are statistically significant, several are particularly noteworthy. First, overweight and self-esteem were not related. This finding contrasts with the findings from Miller and Downey's meta-analysis, (Miller and Downey, 1999) but is consistent the findings of Friedman and Brownell (1995) and Mendelson et al. (1996). However, both BMI and gender were related negatively to body esteem. Also as expected, BMI and gender were associated with personal beliefs that weight is controllable/uncontrollable. Other correlations were consistent with the mediational hypotheses proposed earlier. For instance, self-esteem was correlated negatively with anti-fat attitudes, negative stereotypes of the obese, and thin idealization. Importantly, these correlations (SATAQ, stereotyping, anti-fat attitudes with self- esteem) remained significant $(r \mathrm{~s}=-0.23,-0.28,-0.37$, respectively) when they were conducted without participants whose BMIs were 25 or higher ( 24 is the upper limit for individuals considered "normal" weight).

As outlined in the introductory section, beliefs that obese persons are "failures" whose weight could (andas the prejudice goes-should) be controlled through willpower index ideas about the mobility potential of obese persons (i.e., the beliefs that the obese are members of a social class rather than a social caste). Consistent with our mediational hypotheses, SATAQ scores were related positively to internal attributions for obesity. Somewhat unexpectedly, beliefs in social/environmental causes, such as the amount of food available to obese people, were also linked to self-esteem. To explain this relationship, we propose the following: Because it is likely that the thin ideal is believed (by those who cling to it) to be best achieved through exertions of the will, the antithesis of thinnessobesity-is likely seen as resulting from the failure to exercise sufficient willpower (hence, the relation between the SATAQ and internal causes). This lack of fortitude may, in turn, include a general shortcoming in combating pressures exerted by peers and family members to overeat-hence the correlations from social causes to internal causes, the SATAQ, and negative attitudes toward and stereotypes of obesity.

It may therefore be that those who "blame" obese individuals for their weight status blame them by not only negatively stereotyping them, but also by holding the obese responsible for failing to effectively deal with the social forces that encourage overeating. In other words, the attribution of weight to social causes is an extension of the more general tendency to attribute weight to an individual's character. In support of this proposal, 
partial correlational analyses of the relationships of social cause beliefs to SATAQ, stereotype, and AFA scores indicated that these correlations were no longer significant (largest $r=0.07$ ) when variance associated with internal causes was controlled.

\section{Do Blame Attributions and Beliefs in the Thin Ideal Mediate the Relationship Between Self-esteem and Negative Attitudes toward Obesity?}

The theoretical analysis presented earlier led to the hypothesis that individuals with low self-esteem would negatively stereotype the obese primarily when obesity was considered a social class (as opposed to a social caste). Individuals maintaining this "upward mobility potential" belief system-that obesity is a social category that can be transcended through individual effort-were expected to blame the obese for their physical appearance by attributing obesity to personality shortcomings. These attributional efforts should be more characteristic of those with low self-esteem than of individuals with relatively high self-esteem. It was further hypothesized that one reason for individual differences in internalization of the "thin ideal" is the degree to which an individual identifies with the in-group. Hence, the self-esteem/negative attitudes toward obesity relationship should be mediated by both internal beliefs about the causes of obesity (i.e., "blame" attributions) and in-group identification (indexed by the SATAQ).

To examine these hypotheses, a hierarchical multiple regression analysis of obese stereotypes and negative affective reactions to obesity, with SATAQ, internal causes, and self-esteem scores as predictors, was conducted. Because stereotyping and AFA scores were highly correlated (see Table IV), an initial step was the creation of a composite "negative attitudes toward obesity" (NATO) score (created by first standardizing and then summing AFA and stereotype scores).

SATAQ scores and internal attributions, entered at the first step, together accounted for $30 \%$ of the variance in

Table IV. Hierarchical Regression Analysis of NATO With Internal Attributions, SATAQ, and Self-Esteem as Predictors

\begin{tabular}{llll}
\hline Step & & $R^{2}$ & $\mathrm{~B}$ \\
\hline 1. & Internal causality & & $0.49^{*}$ \\
& SATAQ & & 0.10 \\
2. & Self-esteem & $0.30^{*}$ & -0.13 \\
& & $0.32^{*}$ & \\
\hline
\end{tabular}

$* p<0.001$.
NATO. Consistent with predictions, when self-esteem was entered at the final step, it did not predict unique variance. Examination of the beta values in the table indicates that the belief that obesity is an outcome of characterological flaws, but not thin idealization, mediates the influence of self-esteem on negative attitudes toward obesity.

\section{Do Beliefs in the Value of Thinness and Negative Attitudes Toward Obesity Mediate the Relationship Between Perceived Lack of Control Over Weight and Self-Esteem?}

To examine the hypothesis that the effects of believing that one has little control over weight (PCOW) on self-esteem are mediated by negative attitudes toward obesity and thin idealization, a second multiple regression analysis was conducted. NATO and SATAQ scores, entered at the first step, accounted for $8.7 \%$ of the variance in self-esteem $(p=0.009)$. PCOW scores, entered at the second step, accounted for an additional $2.1 \%$ of variance. Although the overall model was significant ( $p$ $=0.008$ ), only the SATAQ accounted for (marginally) significant variance $(B=-0.19, p=0.055)$; the contributions of neither NATO $(B=-0.17, p=0.074)$ nor PCOW ( $B=-0.15, p>0.10$ ) reached conventional levels of statistical significance. When the same analysis was conducted with SATAQ scores entered at the last step, their contribution continued to border on significance.

Although the total variance in self-esteem that these predictors explained was relatively small (10.8\%), they nonetheless support the hypothesis that perceived lack of control over weight does not, in and of itself, affect self-esteem. Rather, this perception must be coupled with negative attitudes toward obesity and, more importantly, an unrealistic standard-ultrathinness - that is used for self-evaluations.

\section{Do Beliefs in Personal Control Over Weight and the Thin Ideal Mediate the Relationships of Weight and Gender to Body Esteem?}

A multiple regression analysis was conducted to examine whether the link between body esteem and BMI was mediated by SATAQ and PCOW scores. The latter two variables, entered first, accounted for $39 \%$ of the variance on BES ( $p<0.001)$. However, BMI scores accounted for an additional $10 \%$ of variance $(p<0.001)$. All three variables were significant predictors both when BMI scores were entered before SATAQ and PCOW scores and when BMI scores were entered at the second 
step $(B=-0.49,-0.22$, and $-0.34, p<0.01$, for SATAQ, PCOW, and BMI, respectively).

Our primary aim in the next analysis was to determine whether SATAQ and PCOW scores are useful in explaining the link between gender and body esteem. A multiple regression analysis, with PCOW and SATAQ scores entered first, indicated that these variables accounted for $36 \%$ of the variance in body esteem $(p<0.001)$. At the next step, the contribution of participant gender was not significant $(F$ change $<1 ; B=-0.06)$. Examination of the beta values showed that the PCOW $(B=-0.36$, $p<0.001)$ and SATAQ $(B=-0.41, p<0.001)$ independently accounted for significant variance in body esteem.

\section{DISCUSSION}

This investigation examined a number of previously untested hypotheses about the relationships among causal attributions, cultural and personal beliefs about thinness and obesity, attitudes toward and stereotypes of obese persons, and self-esteem. To summarize the principle findings:

(1) Self-esteem and negative attitudes toward obesity are negatively correlated. This relationship, however, appears to be mediated by internal attributions concerning the causes of obesity. Although internalization of the thin ideal was related to both self-esteem and NATO, any effects of thin idealization on self-esteem appear to be subordinated to the effects of internal attributions.

(2) In another set of analyses, the relationship between beliefs that one's weight is controllable and self-esteem was mediated primarily by the extent to which the thin ideal had been internalized. Although negative attitudes toward obesity may also mediate this relationship (recall that the predictive value of NATO to self-esteem approached, but did not attain, significance), additional research examining this possibility is needed.

(3) As expected, gender and body mass were associated with body esteem. Regression analyses indicated the BMI-body esteem relationship is partially, but by no means entirely, mediated by beliefs in the controllability of one's weight and by valuation of the thin ideal. By contrast, the association between gender and body esteem was clearly more indirect. Specifically, women were more likely than men to believe that they did not have control over their weight and to have internalized cultural messages about the importance of thinness to personal success. These two variables, rather than gender per se, explain considerable variance in body esteem.

To varying degrees, each of these findings is consistent with the predictions outlined previously. The most novel contribution is this research concerns two questions that had heretofore not been addressed by researchers interested in the psychosocial correlates of obesity. Specifically, we investigated the "effects" (we use this term cautiously because of the correlational nature of this research) of self-esteem, causal attributions, and thin idealization on negative attitudes toward and stereotypes of obese (self-esteem-> IC + SATAQ->NATO) people and, conversely, the "effects" of negative stereotypes, personal control over weight, and thin idealization on self-esteem (PCOW-> SATAQ + NATO->self-esteem). Concerning the former relationship, from social identity theory we derived the hypothesis that internalization of cultural beliefs about the value of thinness and attributions of blame to the obese for their weight would play roles as mediators. Returning to the ideas discussed in the introductory section, low self-esteem individuals are more likely than those with high self-esteem to (at least attempt to) affiliate with a dominant, high status group in a culture. The implicit goal of these attempts is the "shine in the reflected light" (see Tessor, 1986) of a generalized other, thereby enhancing subjective status.

The attempt to increase self-worth by derogating an out-group, however, is unlikely to be successful if outgroup members cannot be held accountable for their low social standing. It follows that low self-esteem would affect attitudes toward obesity by way of internal attributions for weight status. Postindustrial societies place a premium on an individual's skills, efforts, and abilities - that is, on individualism - as determinants of success and failure and tend to downplay forces (e.g., family background) extraneous to the individual. Relevant to this point is the work of Crandall et al. (2001), who reported that the degree to which obesity is devalued varies between cultures. Specifically, individualistic cultures, such as the United States and Australia, are more prejudiced against obese people than collectivistic cultures, such as India. Although simple categorizations of individualistic and collectivistic cultures have been widely criticized, the Crandall et al. data are generally consistent with the present findings, as those researchers found that the relationship between culture and obese prejudice was mediated by the belief that people are responsible for their weight. 
Thus, where obesity is concerned, low self-esteem individuals need only to apply a general belief systemconcerning the responsibility of individuals in success and failure - to the domain of weight. The (untested) implication of this analysis is that low self-esteem should be related not only to derogation of obese people, but also to the devaluation of other out-groups that, at least in an individual's perceptions, can be held accountable for their low status. For instance, do low self-esteem youths from middle and upper-middle SES backgrounds attribute low SES to the failure of economically impoverished adults to lack of effort? Do low self-esteem youths make similar attributions to groups in oppressed societies (e.g., Palestinians) and affiliate more strongly than high self-esteem individuals with their own cultures?

However, obesity may differ in important ways from other social categories. One factor that may set the obese apart from these other groups is that there exists a clear, omnipresent, and highly unrealistic standard that can be (and is) used to make evaluations. Even if low self-esteem youths do not personally meet the standards for beauty emphasized in Western societies, they can evaluate othersparticularly those who deviate most clearly from the thin ideal-against it. Given its ubiquity, its apparent appeal, and its general acceptance, the thin ideal may be easier than other standards (e.g., race) to apply in the process of making interpersonal judgments.

The thin ideal has multiple functions, however, and often functions more to diminish than to enhance selfesteem (Thompson and Stice, 2001). Our second major finding was that any effects beliefs in personal control over weight have on self-esteem are mediated by thin idealization beliefs and, to a lessor extent, by negative attitudes toward obesity. Only, or primarily, when they devalue overweight individuals and idealize ultrathinness do youths who perceive their own weight as uncontrollable suffer from low self-esteem. Our suggestion is that beliefs in the controllability of weight affect youth's selfesteem in large part when two belief systems have been adopted: That thinness represents a marker for success in our society and that obesity is to be avoided and feared both because of its impact on attractiveness and because of the characterological flaws associated with it. Lack of control means that no amount of personal effort will allow the attainment of the "perfect," highly desirable body and that a "slide" from an acceptable (if not perfect) weight group into a strongly stigmatized status cannot be prevented by acts of will.

The final point of our discussion concerns gender. A well-established finding in the weight and obesity literature is that body image is a greater concern to women than men. The relationship between gender and body esteem is mediated both by beliefs that one's weight is controllable and by degree of internalization of cultural ideas about thinness. Regarding the latter, we advise caution because the version of the SATAQ designed for men and the version designed for women are somewhat different (i.e., the male version emphasizes muscularity more than thinness). Nonetheless, questions remain about why women, more so than men, believe their weight is uncontrollable. One possible reason is that, because women diet more than men and because dieting often does not have its desired effects (and sometimes has effects opposite those it is intended to have), women have a greater empirical basis for believing that their weight cannot be controlled.

In conclusion, this research is an important step toward illuminating the relationships among stereotypes of obesity, self-esteem, and causal attributions. Longitudinal data, as well as research on a wider age range than the young adults we studied, will be important in determining more precisely the nature of causal relationships among these and other variables. Theoretically, we suggest that negative attitudes toward obese individuals are created, to some extent at least, by the (probably unconscious) need for low self-esteem youths to affiliate with a dominant in-group and, subsequently, to increase their subjective status by further derogating an already devalued social group-individuals who are obese.

\section{REFERENCES}

Archibald, A., Graber, J., and Brooks-Gunn, J. (1999). Associations among parent-adolescent relationships, pubertal growth, dieting, and body image in young adolescent girls: A short-term longitudinal study. J. Res. Adolescence, 9: 427-453.

Baumeister, R. F., and Leary, M. R. (1995). The need to belong: Desire for interpersonal attachments as a fundamental human motivation. Psychol. Bull. 117: 497-527.

Bell, S. K., and Morgan, S. B. (2000). Children's attitudes and behavioral intentions toward a peer presented as obese: Does a medical explanation for obesity make a difference? J. Pediatr. Psychol. 25: $137-145$.

Bigler, R. S., Jones, L. C., and Lobliner, D. B. (1997). Social categorization and intergroup attitudes in children. Child Dev. 68: 530-543.

Boyer, E. (1986, December). Transition from school to college. Phi Delta Kappan, 293-297.

Centers for Disease Control (2002). Prevalence of overweight among children and adolescents: United States, 1999. Retrieved from www.cdc.gov/nccdphp/dnpa/obesity/trend/index.htm

Crandall, C. S. (1994). Prejudice against fat people: Ideology and selfinterest. J. Pers. Soc. Psychol. 66: 882-894.

Crandall, C. S., and Biernat, M. R. (1990). The ideology of anti-fat attitudes. J Appl. Soc. Psychol. 20: 227-243.

Crandall, C. S., D’Anello, S. D., Sakalli, N., Wieczorkowski, G., and Feather, N. T. (2001). An attribution model of prejudice: Anti-fat attitudes in six nations. Pers. Soc. Psychol. Bull. 27: 30-37.

Crocker, J., Cornwell, B., and Major, B. (1993). The stigma of overweight: Affective consequences of attributional ambiguity. J. Pers. Soc. Psychol. 64: 60-70. 
Crystal, D. S., Watanabe, H., and Chin, W. (1997). Intolerance of human differences: A cross-cultural and developmental study of American, Japanese, and Chinese children. J. Appl. Dev. Psychol. 18: 149167.

Crystal, D. S., Watanabe, H., and San Chen, R., (2000). Reactions to morphological deviance: A comparison of Japanese and American children and adolescents. Soc. Dev. 9: 40-62.

Friedman, M. A., and Brownell, K. D. (1995). Psychological correlates of heavyweight: Moving to the next generation of research. Psychol. Bull. 117: 3-20.

Gable, S., and Lutz, S. (2000). Household, parent, and child contributions to childhood obesity. Fam. Relat. 49: 293-300.

Harter, S. (1985). Competence as a dimension of self-evaluation: Toward a comprehensive model of self-worth. In Leahy, R. (ed.), The Development of Self. Academic Press, New York, pp. 55122.

Harter, S. (1993). Causes and consequences of low self-esteem in children and adolescents. In Baumeister, R. F. (ed.), Self-Esteem: The Puzzle of Low Self-Regard. Plenum, NY, pp. 87-116.

Heinberg, L. J., Thompson, J. K., and Stormer, S. (1995) Development and validation of the Sociocultural Attitudes Towards Appearance Questionnaire (SATAQ). Int. J. Eat. Disord. 17: 8189.

Hewstone, M., Islam, M. R., and Judd, C. M. (1993). Models of crossed categorization and intergroup relations. J Pers. Soc. Psychol. 64: 779-793.

Hewstone, M., Jaspars, J., and Lalljee, M. (1982). Social representations, social attribution, and social identity: The intergroup images of "public" and "comprehensive" schoolboys. Eur. J. Soc. Psychol. 12: $241-269$.

Jessor, R., Van Den Bos, J., Vanderryn, J., Costa, F., and Turbin, M. (1995). Protective factors in adolescent problem behavior: Moderating effects and developmental change, Dev. Psychol. 31: 923933.

Klaczynski, P. A. (1991). Sociocultural myths and occupational attainment: Educational influences on adolescents' perceptions of social status. Youth Soc. 22: 448-467.

Klaczynski, P. A. (2000). Motivated scientific reasoning biases, epistemological beliefs, and theory polarization: A two-process approach to adolescent cognition. Child Dev. 71: 1347-1366.

Klaczynski, P. A., and Aneja, A. (2002). The development of quantitative reasoning and gender biases. Dev. Psychol. 38: 208-221.

Lerner, R. M., and Korn, S. J. (1972). The development of body-build stereotypes in males. Child Dev. 43: 908-920.

Lewis, R. J., Cash, T. F., Jacobi, L., and Bubb-Lewis, C. (1997). Prejudice toward fat people: The development and validation of the Antifat Attitudes Test. Obes. Res. 5: 297-307.

Mendelson, B. K., White, D. R., and Balfour, L. (1995). The BodyEsteem Scale for Adolescents and Adults. Unpublished manuscript, Concordia University, Montreal, Quebec.

Mendelson, B. K., White, D. R., and Mendelson, M. J. (1996). Selfesteem and body esteem: Effects of gender, age, and weight. $J$. Appl. Dev. Psychol. 17: 321-346.

Miller, C. T., and Downey, K. T. (1999). A meta-analysis of heavyweight and self-esteem. Pers. Soc. Psychol. Rev. 3: 68-84.
Nestle, M. (2002). Food Politics. University of California Press, Los Angeles, CA.

Osgood, D. W., Wilson, J., O'Malley, P., Bachman, J., and Johnston, L. (1996). Routine activities and individual deviant behavior. Am. Sociol. Rev. 61: 635-655.

Peterson, A. (1988). Adolescent development. Annu. Rev. Psychol. 39: 583-607.

Pettigrew, T. F. (1979). The ultimate attribution error: Extending Allport's cognitive analysis of prejudice. Pers. Soc. Psychol. Bull. 5: 461-476.

Pierce, J., and Wardle, J. (1997). Cause and effect beliefs and self-esteem of overweight children. J. Child Psychol. Psychiatry, 38: 645-650.

Quinn, D. M., and Crocker, J. (1999). When ideology hurts: Effects of belief in the Protestant ethic and feeling overweight on the psychological well-being off women. J. Pers. Soc. Psychol. 77: 402-414.

Rosenburg, M. (1979). Conceiving the Self. Basic Books, NY.

Simmons, R. G., and Rosenberg. M. (1971). Functions of children's perceptions of the stratification system. Am. Sociol. Rev. 36: 235249

Simon, B., and Brown, R. (1987). Perceived intragroup homogeneity in minority-majority contexts. J. Pers. Soc. Psychol. 53: 703-711.

Staffieri, J. R. (1967). A study of social stereotype of body image in children. J. Pers. Soc. Psychol. 7: 101-104.

Steinberg, L. (2002). Adolescence (6th edn.). McGraw-Hill, Boston, MA.

Tajfel, H. (1982). Social psychology of intergroup relations. Annи. Rev. Psychol. 33: 1-39.

Tajfel, H., and Turner, J. C. (1979). An integrative theory of intergroup conflict. In Austin, W. G., and Worchel, S. (eds.), The Social Psychology of Intergroup Relations. Brooks/Cole, Monterey, CA, pp. 33-47.

Taylor, D. M., Moghaddam, F. M., and Bellerose, J. (1989). Social comparison in an intergroup context. J. Soc. Psychol. 129: 499515 .

Tesser, A. (1986). Some effects of self-evaluation maintenance on cognition and action. In Sorrentino, R. M., and Higgins, E. T. (eds.), Handbook of Motivation and Cognition. Guilford, NY, pp. 435464.

Thompson, J. K., and Stice, E. (2001). Thin-ideal internalization: Mounting evidence for a new risk factor for body-image disturbance and eating pathology. Curr. Dir. Psychol. Sci. 10: 181-183.

Thurstone, T. G. (1962). Primary Mental Abilities for Grades 9-12. Science Research Associates, Chicago.

Turner, R. H. (1960). Sponsored and contest mobility and the school system. Am. Sociol. Rev. 25: 855-867.

Weiner, B., Perry, R. P., and Magnusson, J. (1988). An attributional analysis of reactions to stigmas. J. Pers. Soc. Psychol. 55: 738748

Willis, S., and Schaie, K. W. (1996). Psychometric intelligence and aging. In Blanchard-Fields, F., and Hess, T. M. (eds.), Perspectives on Cognitive Change in Adulthood and Aging. McGraw-Hill, New York, pp. 293-322.

Wing, R. R., and Jeffrey, R. W. (1999). Benefits of recruiting participants with friends and increasing social support for weight loss and maintenance. J. Consulting Clin. Psychol. 67: 132-138. 